\title{
The EMMA Multimedia Conference Service
}

\author{
Nils Björkman ${ }^{1}$, István Cselényi ${ }^{2}$, Alexander Latour-Henner ${ }^{3}$, Gergely Záruba ${ }^{2}$ \\ ${ }^{1}$ Center for ATM Research At Telia, Dept. of Network Research, Telia Research AB \\ Rudsjöterrassen 2. S-13680 Haninge, Sweden, Phone: +468 7075562, E-mail: \\ nils.p.bjorkman@telia.se \\ ${ }^{2}$ High Speed Networks Laboratory, Dept. of Telecommunications. and Telematics, TU Budapest \\ Stoczek u. 2., H-1111 Budapest, Hungary, Phone: +36 1 4633110, E-mail: zaruba@inf.bme.hu \\ ${ }^{3}$ Telia Instruments, Telia AB, Vitsandsgatan 9, Building B. \\ S-12386 Farsta, Sweden, Phone: +46 871325 39, E-mail: alexander.a.latour-henner@telia.se
}

\begin{abstract}
The complexity and diversity of novel telecommunication services require experimental systems for the exploration of research areas such as resource allocation and management.

The aim of the paper is to introduce a multimedia conferencing service and to summarize its main benefits. This service is based on an experimental system, which deals with research issues both on the network and terminal side and which is adequate for quantitative and subjective evaluation of teleservices.

An object-oriented model describes the parties involved in the session, the allocated network and terminal resources and the media used for communication. The objects of this model are created and updated by a high level service management protocol. The centralized resource management scheme ensures QoS for any kind of multimedia services. An other benefit of this system is that the control of the service can be done by naive users.
\end{abstract}

\section{Introduction}

The EMMA-SIGNE system follows the traditional, network-oriented service management approach, i.e. it aims full control of terminal and network resources $[1,2]$. The actual teleservice session, called Service Instance, is described by a universal, object oriented model which represents the parties involved in the session, the media they are using to communicate, the network resources e.g. ATM connections and the relations of these objects. The whole, global view of the
Service Instance is stored in the network while partial views are stored as Local Views of Service Instance in the EMMA terminals. During the set-up and the lifecycle of the session, a high level protocol is used for starting, modifying and stopping the objects of these models, i.e. the represented terminal and network resources. This service management protocol is based on TCP/IP.

Section two gives a general description of the EMMASIGNE experimental system. Section three introduces the multimedia conference service and section four enumerates the benefits of the system and concludes the paper.

\section{The Experimental System}

The main structural parts of the EMMA-SIGNE experimental system and their relationship are shown in figure 1 . The components are playing an important role in the service managernent and are introduced in the followings.

There are three main entities in the experimental system:

- the provider of the network

- the provider of the conferencing service

- the terminal used for the communication

The name of the first entity is Signaling Emulator (SIGNE) [3] and the second and third belong to the Experimental Multimedia Middleware for ATM (EMMA) [4]. 


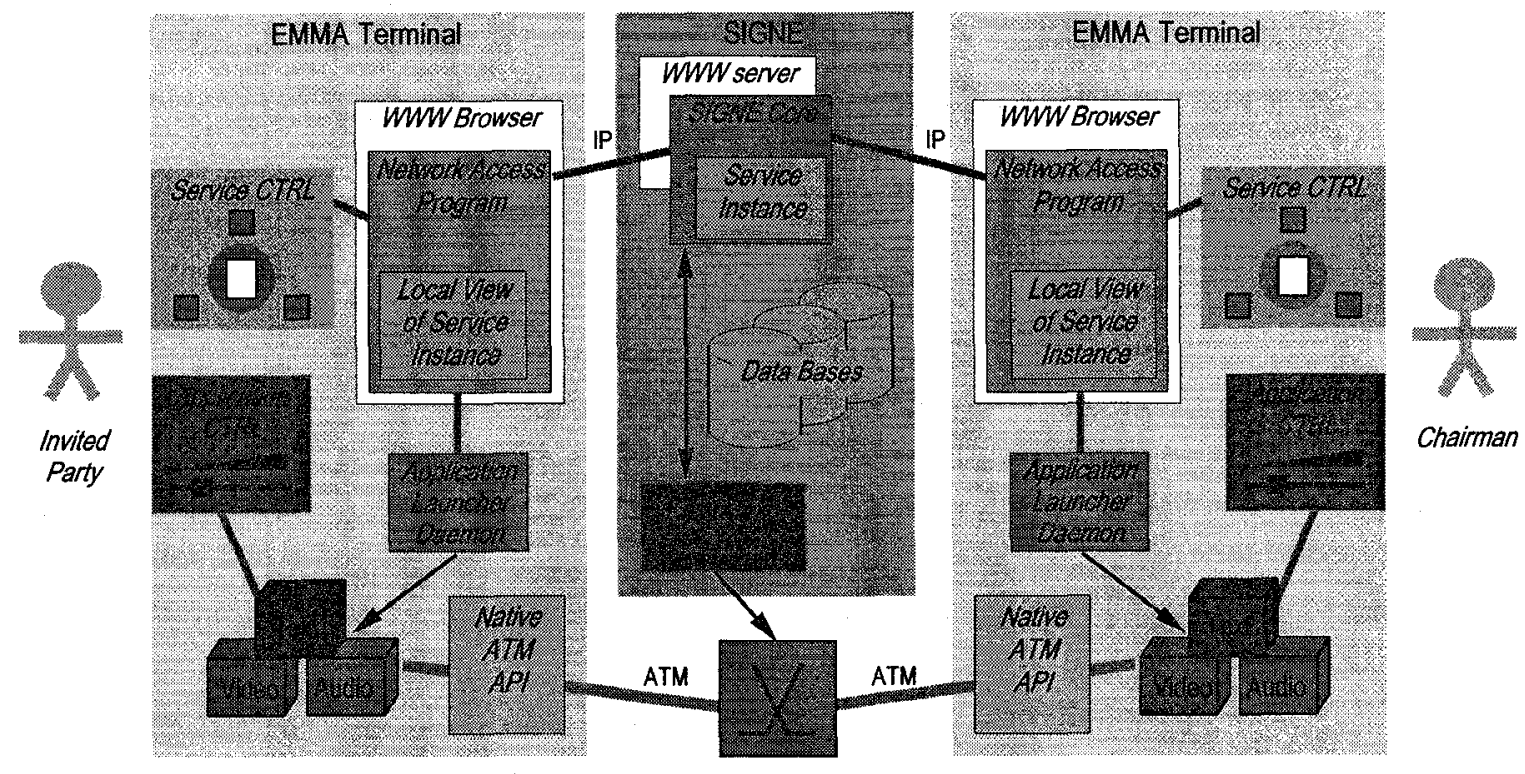

Figure 1. The Multimedia Conference Based on the EMMA-SIGNE Experimental System

\subsection{Session Set-Up}

Naive users can join to the system by surfing with their $W W W$ Browser onto the homepage provided by SIGNE. After registration and selection of the service (and eventually the service provider), they can order a new session or modify the ongoing session by a Java-based Service Control interface. They can give their quality requirements in user-friendly terms (like high, medium or low resolution, big, medium or small picture size, etc.), which will be mapped to specific resource parameters by the system. SIGNE Core collects the quality requirements of each party, allocates the network resources through the Network Control unit according to the commonly accepted quality level and starts the session by sending a confirmed state message to the terminals' Network Access Program (NAP) which uses the Application Launcher Daemon (AppLD) to start the Application Building Blocks (ABB).

\subsection{Flexible Service Description}

There is a general model for telecommunication services, provided on the EMMA-SIGNE platform. This model is based on the results of RACE R2044 Multiservice Applications Governing Integrated Control (MAGIC) project [5], which has been extended by Telia. Figure 2. presents a model of the generic Telecommunication Service Description, the objects and their relationships.

There are optional and mandatory service objects. An object can be realized if every mandatory object has been built up in the hierarchy below it. Service Instance contains attributes to describe the overall operation of the service. The Party object represents the behavior of the service user.

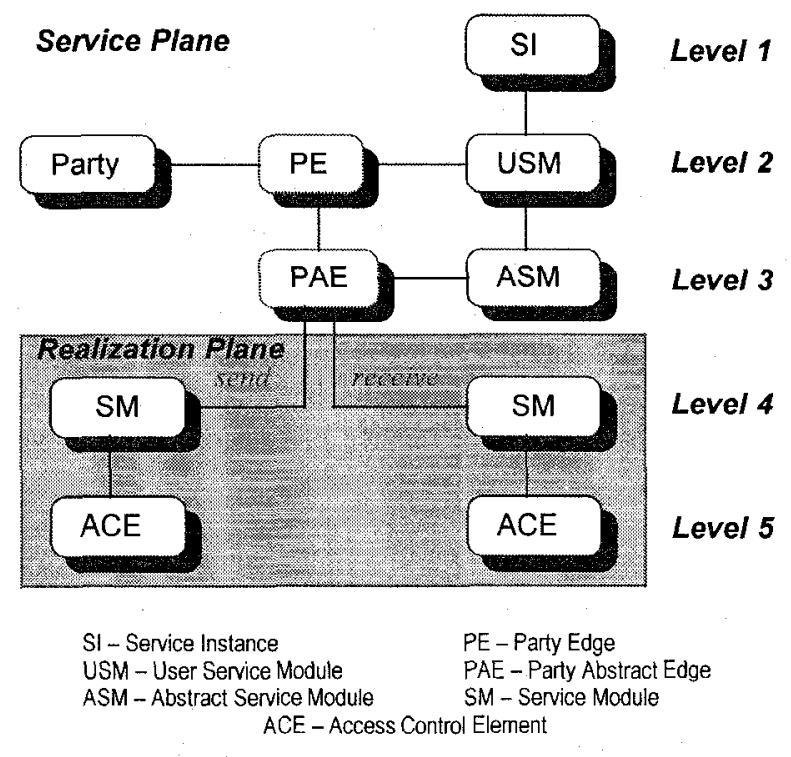

Figure 2. Telecommunication Service Description

User Service Module represents the complex media involved in the service session. The Party Edge defines the mapping between the Parties and the USMs that comprise the service. Abstract Service Module represents the basic information types that may be used in the service. The USMs consist of one or more ASMs. The Party Abstract Edge is used to describe the relationship between the ASMs and the PE so it maps the direction of information exchange - send or receive. Service Module represents the protocols used to 
implement a basic information type of a multimedia service. Protocols from the ATM Adaptation Layer to the presentation layer are specified, so the SM is used to implement the service indicated in the ASM. Access Control Element defines the characteristics of the bearer connection that is used to transport the information of the SMs across the network. It encompasses the user information protocols in the protocol stack of the Physical Layer and the ATM Layer.

\subsection{Communication with the Network}

A new, high level signaling protocol-family had to be developed for EMMA, namely the Telecommunication Service Protocol (TSP). It is operating over TCP/IP and implements the signaling required for the establishment and control of multimedia services.

The mostly used member of this family is the TSP/SIC (Telecommunication Service Protocol/Service Instance Control) message. There are four possible types of this message (as shown in figure 3):

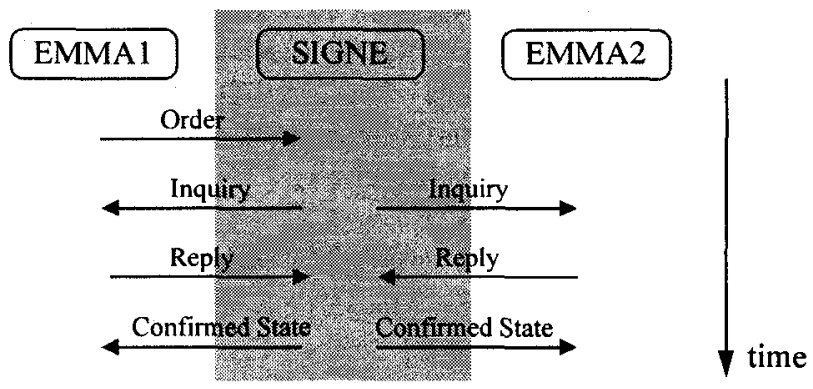

Figure 3. The Sequence of the TSP/SIC Messages

- Order, this message goes from EMMA to SIGNE, If a terminal wants to change the Service Instance (which also means that it wants to establish a new SI e.g. a conference).

- Inquiry is the answer of SIGNE to the initiator EMMA-terminal about the acceptance of the changes of the Order message. Furthermore This message is also sent to the other EMMA-terminals which are asked to be involved by the initiator.

- Reply is sent to the NAP from all of the involved EMMA-terminals which includes the acceptation or rejection for each of the telecommunication service objects included in the Inquiry message.

- Confirmed State. SIGNE checks all the incoming Reply messages and sends the final states of the telecommunication service objects to all of the EMMA-terminals, so the consistency is ensured.

\subsection{The EMMA Terminal}

EMMA is realized on a general purpose workstation [6]. The main structural parts of the terminal are:
- Network Access Program for service consumer and provider in order to make the easy use of the User Applications possible.

- User Applications (UA) containing the Application Building Blocks which provide the multimedia services to the users. The ABBs can access the ATM network through the specially developed EMMA component: the Native ATM Programming Interface [7].

The name of the ABB must be explained more: it has got its name because the concept is to make a program which can be recycled for all the multimedia applications which use the same representation media (for example a video conference service and a video demand service can use the same video- $A B B$ ).

Since there are two main parts which have to communicate, we also need a possible high-level protocol between NAP and the UAs.

\subsection{Communication with the User}

The communication with the user is a task of the NAP. There is a JAVA application downloaded from the SIGNE server for each of the provided services. This user friendly interface is called Service Control. The Service Control's task is to control the network and terminal resources, so that even naiv users can use the services. In a case of video conference, for instance, the user does not have to say anything about the coding schemes, required bandwidth, the user only has to give a quality value like good, medium or bad for the picture transmission. An other task of the Service Control is to inform the user about the costs and the eventually errors occurred at setting-up or using time.

\section{EMMA Multimedia Conference}

The first multimedia service which is made to test the EMMA/SIGNE concept is the EMMA Multimedia Conference - EMMC. This is a multiparty conference tool which includes audio and video conference and whiteboard.

\subsection{Service Description}

The $S I$ is called EMMC. There are two possible kinds of USMs: Videophone and Whiteboard. Three ASMs are allowed: Audio, Video (which are child-objects of the Videophone USM) and Whiteboard (which is a child-object of the Whiteboard USM). The Party type can be chairman or member. Chairman is the one who can change the objects in the conference (usually the initiator of the conference), so add/drop parties and modify network related settings. An other attribute of this object is the terminal name on which it is 
connected. The $P E$ and $P A E$ objects describe the mapping of the USMs, ASMs, Parties and SM to each other. Since there is only one $A C E$ belonging to one $S M$, there is no need to separate strongly these two objects. These objects have parameters like direction, the used VP (Virtual Path) and VC (Virtual Channel) pairs of the ATM connection. Furthermore there are ASM dependent parameters e.g. coding type, number of channels etc. in a case of an audio-ASM.

\subsection{Communication with the User}

A JAVA based user interface has been developed to handle the communication between NAP. With the help of this the user can see the current state of the conference and set the network related service parameters. The graphical user interface (GUI) can be seen on figure 4.

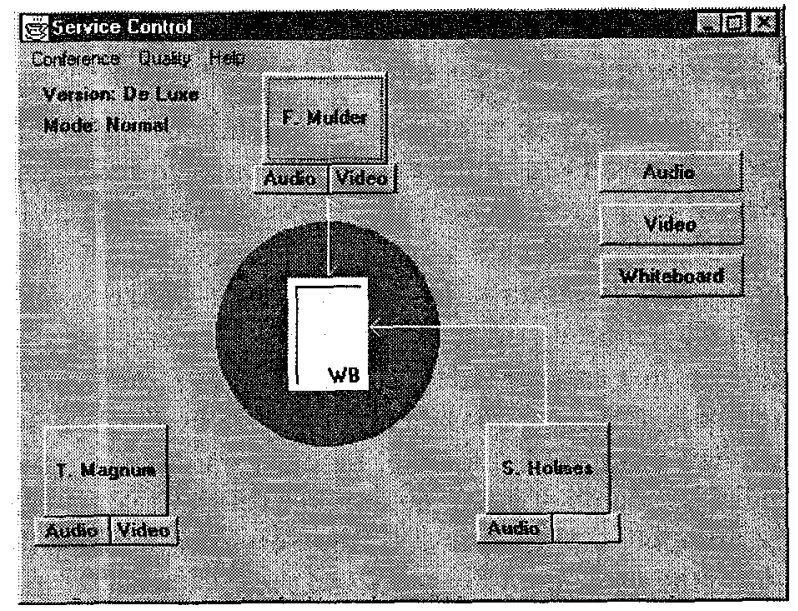

Figure 4. The Service Control of EMMC

According to figure 4, all of the parties are using audio conference, agent Mulder and P.I. Magnum have video connection and $\mathrm{Mr}$. Holmes and agent Mulder use the whiteboard.

On the left side there are the buttons Audio, Video and Whiteboard, modifying the media used in the conference.

\subsection{Communication inside of EMMA}

A communication is needed between the Network Access Program and the User Applications to realize the conference which has been created by NAP and SIGNE. The User Applications (UAs) have the running Application Launcher Daemon on each of the EMMA multimedia terminals. This daemon watches a given TCP port. The NAP will contact the AppLD through this port.

There are three kinds of messages, which are exchanged between the NAP and the Application Launcher
Daemon (AppLD) according to the Order, Inquiry and Confirmed State primitives of the TSP/SIC protocol.

\section{The $\boldsymbol{U A}$-Order and $\boldsymbol{U} A-O r d e r-R e p l y$ Message}

The UA-Order message comes from NAP to the AppLD. The message is used in the ordering phase for negotiation about the creation of a new USM or ASM entity. This message indicates to the daemon, which application it has to launch. The daemon launches these processes and make them check whether the required resources are ready, if they are then the processes have to reserve these resources (like the audio device in case of an audio-ASM). The launched processes have to return an answer whether they succeed to fulfill the request before. The AppLD will collect the negative and/or positive answers and will sent it back in the $U A$ -Order-Reply message.

\section{The $U A$-Inquiry and $U A$-Inquiry-Reply Message}

The UA-Inquiry message has to come from NAP at every TSP/SIC inquiry, that NAP receives. It contains information which is relevant to the USM and ASM objects. The message is nearly the same as the $U A$ Confirmed-State message. The AppLD has to answer with an UA-Inquiry-Reply message after the negotiation with the processes it has launched.

\section{The UA-Confirmed State Message and Its Answer}

The UA-Confirmed State message is used to send the parts of the Local View Of Service Instance from NAP to the AppLD. The AppLD will build a hierarchical object inside itself. Also the format of the message is hierarchical, furthermore it is a simplified version of a TSP/SIC-Confirmed State message. After the daemon receives this message and builds the object, it has to inform its children and negotiate with them. Error values are to collected and to sent back to NAP using the UA-Confirmed State-Reply message.

\subsection{Application Building Blocks}

The Multimedia Conference service uses three kind of ABBs for presenting video, audio and white board information to the user. The audio-ABB handles the audio $\mathrm{I} / \mathrm{O}$ devices of the multimedia terminal (e.g. microphone, speaker, line in/out), sends/receives, mixes and codes/encodes the audio data streams from/to the other terminals. The video-ABB makes the same for the video data streams, so the handling of the cameras and the displaying of these streams are included. The whiteboard-ABB uses a special developed whiteboard protocol which ensures the consistency between the users and presents the data on the display. 


\section{Conclusion}

The EMMA-SIGNE experimental system has many benefits from the service management's point of view. In spite of the dynamic routing paradigm, where unlimited network resources are assumed and the quality demands supposed to be fulfilled in a dynamic and flexible manner, our system utilizes the central view of the service for full control of network and terminal resources. This way not only a "best effort" can be done but the quality of service can be ensured just as it was required by the users.

The key concepts are to

- make a generic conferencing system which can be controlled by naive users,

- develop protocols for transmitting data about distributed multimedia services, so all the participants have a consistent view of the service at time,

- make a testing tool for broadband networks, especially for ATM,

- make the basic idea grow to a globally used and accepted network management system.

The novel features include:

- hot-billing and pricing of multimedia teleservices,

- distributed real-time multimedia transmission over ATM,

- a JAVA written user interface which can be accessed with a WWW browser,

- developing building blocks, which can be re-used by more applications using the same representation media.

The first phase of the project is close to the end and the range of possible experiments is wider and wider every day.

\section{Acknowledgements}

The reported research activities are made in the joint research program between the CARAT Laboratory of Telia Research in Sweden and the High Speed Networks Laboratory of the Department of Telecommunication and Telematics, Technical University of Budapest in Hungary. We would like to thank our colleagues in Sweden and in Hungary for the help during the design and implementation of this system.

\section{References}

[1] I. Cselényi, C. Gisgård, P. Haraszti, A. LatourHenner, I. Szabó, G. Záruba „Multipurpose Middleware For Broadband Multimedia Applications", ConTEL Broadband \& Multinedia WS, Zagreb, Croatia, November 11-12, 1996

[2] D. Saha, D. Kandlur, T. Barzilai, Z. Shae, M. Willebeek-LeMair „A Video Conferencing Testbed on ATM: Design, Implementation and Optimizations", IEEE Conference on Multimedia Computing and Systems, Washington DC, May 1995

[3] C. Gisgård „SIGNE - Signaling Emulator", Fasrapport, Telia Instrument, Telia AB, 1993.

[4] I. Cselényi, I. Szabó, P. Haraszti, N. Björkman, C. Gisgård „A Versatile Multimedia Platform”, IASTED Int. Conference on Applied Informatics, Innsbruck, Austria, February 17-20, 1997

[5] RACE Project R2044 „MAGIC- Multiservice Applications Governing Integrated Control", Service Description Framework and B-ISDN Service Descriptions, 3rd Deliverable, EC, 1992.

[6] I. Cselényi, I. Szabó, G. Záruba „EMMA Teleconference Version 1.0", High Speed Networks Laboratory Formal Meeting, Budapest, December 1996

[7] ATM Forum Technical Committee "Native ATM Services: Semantic Description", 1995 